\title{
Shape and Size Dependent Electronic Properties of GaAs/AlGaAs Quantum Dots
}

\author{
Dharmendra Kumar, C.M.S. Negi, K. Saral Gupta and Jitendra Kumar
}

\begin{abstract}
We have theoretically investigated the effect of shape anisotropy and size on electronic structure of GaAs/AlGaAs quantum dots (QDs). The quantum dot is modeled by anisotropic parabolic confinement potential in the plane perpendicular to the growth direction while the confinement along the growth direction is modeled as quantum well potential. The Luttinger Hamiltonian formulation has been used to account for the valence subband mixing. The electronic structure is calculated by numerical diagonalization of Luttinger Hamiltonian using the harmonic oscillator basis functions. The calculations for hole energies and transition energies have been carried out over wide range of size and shape of QDs. The results show that transition energy of $Q D S$ decreases with the height of QDs. Significant variation in the hole energy is observed with the change in anisotropy. We also observe that shape anisotropy and mixing have significant effect on the energy states.
\end{abstract}

Keywords--- GaAs/AlGaAs, Self-Assembled Quantum Dot, Luttinger Hamiltonian

\section{INTRODUCTION}

$\mathrm{Q}$ UANTUM dots have been the subject of increased research due to interesting physical properties and promising applications, however it is crucial to understand their electronic structure for optoelectronics application.

Semiconductor nanostructures e.g. quantum wells, quantum wires \& quantum dots (QDs) are undergoing extensive research in aspects of nanotechnology application. Wide range of application have been found such as Lasers [12], LEDs [3] and single photon light sources [4].

Semiconductor quantum dots exhibit electronic and optical properties quite different from those of the bulk semiconductors, as the quantization of all degrees of freedom results in atom like discrete set of energy levels. The electronic structure of the QD can be manipulated by varying the shape, size, and number of electrons associated with it. Size and shape anisotropies play key role in determining the

Dharmendra Kumar, Department of Electronics Engineering, Indian School of Mines, Dhanbad Jharkhand, India $826004 . \quad$ Email:dharmendra0127@gmail.com

C.M.S. Negi, Department of Electronics Banasthali Vidyapith, Rajasthan 304022. E-mail:echandru2001@yahoo.com

K. Saral Gupta, Department of Physics, Banasthali Vidyapith, Rajasthan 304022.E-mail: saral.kumargupta@gmail.com

Jitendra Kumar, Department of Electronics Engineering, Indian School of Mines, Dhanbad, Jharkhand, India 826004. E-mail:jitenkg@rediffmail.com transition energies in a QD and strongly influence its optical response.

GaAs/AlGaAs quantum dots can be fabricated using various techniques like metal organic chemical vapour deposition [5], modified droplet epitaxy [6], etc.

A commonly used method to produce QDs is the StranskiKrastanov (SK) technique. In this technique, material is deposited epitaxially onto a substrate to which it is not lattice matched. Due to the mismatch, the deposited material spontaneously forms nanometer scale islands. SK QDs are strained and significant intermixing usually occurs during island formation [7-10], the used technique and parameters are decided the shape and size of the quantum dots.

In this paper, we have used k.p model for the calculation of the electronic structure of QDs. k.p model is one of the most widely adopted approaches due to its simplicity and accuracy for modeling the band structure near the first Brillion zone center [11-15].

\section{THEORETICAL FORMULATION}

In the present work, we have considered a 4X4 Luttinger Hamiltonian for describing the valance band structure of GaAs/AlGaAs; we choose the z-direction to be the crystal growth direction for GaAs/AlGaAs quantum dot with elliptical cross section in the $(x-y)$ plane. We have taken in-plane $(x-y)$ confinement potential.

$V_{x y}(x, y)=\frac{1}{2} \alpha_{x} x^{2}+\frac{1}{2} \alpha_{y} y^{2}$

where, $\alpha_{x}$ and $\alpha_{y}$ are the force constants that determine the strength of confinement along $x$ and y directions, respectively. Also we assume finite well confinement potential along $\mathrm{z}$ direction. Energies in $\mathrm{z}$ direction for different dot height are numerically obtained using transcendental equation [16].

Without the off-diagonal elements the wave function in the $x-y$ plane would be the harmonic oscillator wave functions

$\phi_{n_{x}, l h}{ }^{(x),} \phi_{n_{y}, l h}{ }^{(y),} \phi_{n_{x}, h h}{ }^{(x),} \phi_{n_{y}, h h}{ }^{(y)}$

where, $\mathrm{n}_{x}$ and $\mathrm{n}_{y}$ are quantum numbers in $x$ and $y$ direction respectively. The form of normalized wave functions is given by

$\phi_{n_{x}}(x)=\left(2^{n_{x}} n_{x} ! \sqrt{\pi} \lambda_{x}^{i}\right)^{-1 / 2} \exp \left(-\frac{1}{2}\left(\frac{x}{\lambda_{x}^{i}}\right)\right) H_{n}\left(\frac{x}{\lambda_{x}^{i}}\right)$ 
where,

$$
\lambda_{x}^{i}=\sqrt{\frac{\hbar}{m \omega_{x}^{i}}},
$$

and $\mathrm{i}=\mathrm{lh}, \mathrm{hh}$

The wave functions in the envelope function approximation for the conduction band can be written as

$\psi_{m s}^{c}(r)=f^{c}(r) u_{m s}^{c}(r)$

Where $u_{m_{s}}^{c}$ are the two spin degenerate states $(\mathrm{s}=1 / 2$, $\left.m_{s}= \pm 1 / 2\right)$ bulk Bloch functions at the bottom of the conduction band and $f^{c}(r)$ are the envelope functions. In the valence band we have two spin degenerate $\mathrm{hh}$ and $\mathrm{lh}$ states, and the corresponding wave functions are given by [17].

$\psi^{v}(r)=\sum_{m} f^{v}(r) u_{m}^{v}(r)$

Here $u_{m_{j}}^{v}$ are the Bloch functions at the top of the valance band with $m_{\mathrm{j}}=+3 / 2$ and $-3 / 2$ for heavy holes and $\mathrm{m}_{\mathrm{j}}=+1 / 2$ and $1 / 2$ for the lh states.

The Bloch wave functions for these energy states can be written as [18].

$$
\begin{aligned}
& \left|\frac{3}{2}, \frac{3}{2}\right\rangle=-\frac{1}{\sqrt{2}}[|X \uparrow\rangle+i|Y \uparrow\rangle] \\
& \left|\frac{3}{2}, \frac{1}{2}\right\rangle=-\frac{1}{\sqrt{6}}\left[|X \downarrow\rangle+i|Y \downarrow\rangle+\sqrt{\frac{2}{3}}|Z \uparrow\rangle\right] \\
& \left|\frac{3}{2},-\frac{1}{2}\right\rangle=\frac{1}{\sqrt{6}}\left[|X \uparrow\rangle+i|Y \uparrow\rangle+\sqrt{\frac{2}{3}}|Z \downarrow\rangle\right] \\
& \left|\frac{3}{2},-\frac{3}{2}\right\rangle=\frac{1}{\sqrt{2}}[|X \downarrow\rangle-i|Y \downarrow\rangle] \\
& \left|\frac{1}{2}, \frac{1}{2}\right\rangle=-\frac{1}{\sqrt{3}}[|X \downarrow\rangle+i|Y \downarrow\rangle+|Z \uparrow\rangle] \\
& \left|\frac{1}{2}, \frac{1}{2}\right\rangle=-\frac{1}{\sqrt{3}}[-|X \downarrow\rangle+i|Y \downarrow\rangle+|Z \downarrow\rangle]
\end{aligned}
$$

Luttinger Hamiltonian matrix used in our calculation is given by

$$
H=\left[\begin{array}{cccc}
H_{h h} & b & c & 0 \\
b^{\dagger} & H_{l h} & 0 & -c \\
c^{\dagger} & 0 & H_{l h} & b \\
0 & -c^{\dagger} & b^{\dagger} & H_{h h}
\end{array}\right]
$$

where,

$$
\begin{aligned}
& H_{h h}=-\frac{\hbar^{2}}{2 m 0}\left[\left(\gamma_{1}-2 \gamma_{2}\right) \frac{d^{2}}{d z^{2}}+\left(\gamma_{1}+\gamma_{2}\right)\left(\frac{d^{2}}{d x^{2}}+\frac{d^{2}}{d y^{2}}\right)\right], \\
& H_{l h}=-\frac{\hbar^{2}}{2 m_{0}}\left[\left(\gamma_{1}+2 \gamma_{2}\right) \frac{d^{2}}{d z^{2}}+\left(\gamma_{1}-\gamma_{2}\right)\left(\frac{d^{2}}{d x^{2}}+\frac{d^{2}}{d y^{2}}\right)\right],
\end{aligned}
$$

$$
\begin{aligned}
& b=\frac{\sqrt{3} \hbar^{2}}{2 m_{0}} \gamma_{2} \frac{d}{d z}\left(\frac{d}{d x}-i \frac{d}{d y}\right), \\
& c=\frac{\sqrt{3} \hbar^{2}}{2 m_{0}} \gamma_{3}\left(\frac{d}{d x}-i \frac{d}{d y}\right)^{2} .
\end{aligned}
$$

In the above equations $\gamma_{1}, \gamma_{2}, \gamma_{3}$ are the Luttinger parameters. We can write down the matrix elements as

$H_{h h}=\left[E_{z}^{h h}+\hbar \omega_{x}^{h h}\left(n_{x}+\frac{1}{2}\right)+\hbar \omega_{y}^{h h}\left(n_{y}+\frac{1}{2}\right)\right] \delta_{n_{x}, n_{x}}{ }^{\prime} \delta_{y^{\prime}}, n_{y}{ }^{\prime}$

$H_{l h}=\left[E_{z}^{l h}+\hbar \omega_{x}^{l h}\left(n_{x}+\frac{1}{2}\right)+\hbar \omega_{y}^{l h}\left(n_{y}+\frac{1}{2}\right)\right] \delta_{n_{x}, n_{x}{ }^{\prime} \delta_{n_{y}}, n_{y}{ }^{\prime}}$

where,

$$
\begin{aligned}
& \omega_{x}^{h h(l h)}=\sqrt{\frac{\alpha_{x}}{m_{h h(l h)}}}, \\
& \omega_{y}^{h h(l h)}=\sqrt{\frac{\alpha_{y}}{m_{h h(l h)}}}
\end{aligned}
$$

and $E_{z}^{h h(h)}$ is the energy of hh (lh) state due to zconfinement.

Here hh and $\mathrm{lh}$ frequencies are given below

$\omega_{x}^{h h}=\left(\omega_{0}+\Delta \omega\right) \sqrt{\gamma_{1}+\gamma_{2}}$

$\omega_{y}^{h h}=\left(\omega_{0}-\Delta \omega\right) \sqrt{\gamma_{1}+\gamma_{2}}$

$\omega_{x}^{l h}=\left(\omega_{0}+\Delta \omega\right) \sqrt{\gamma_{1}-\gamma_{2}}$

$\omega_{y}^{l h}=\left(\omega_{0}-\Delta \omega\right) \sqrt{\gamma_{1}-\gamma_{2}}$

We define the anisotropy parameter A as

$A=\frac{\Delta \omega}{\omega_{0}}$

where $\omega_{0}$ is the isotropic frequency and $\Delta \omega$ is the difference arising due to anisotropy; $\Delta \omega>0$ or $\mathrm{A}>0$ means stronger confinement in $\mathrm{x}$-direction and $\Delta \omega<0$ or $\mathrm{A}<0$ means stronger confinement in y direction.

Hence we get diagonal elements of the heavy hole and the light hole as.

$$
\begin{aligned}
& \left.H_{h h}=\left[E_{z}^{h h}+\hbar \omega_{0}^{h h} \sqrt{\gamma_{1}+\gamma_{2}}\left(\left(n_{x}+n_{y}\right)+A\left(n_{x}-n_{y}\right)+1\right)\right)\right] \delta_{x^{\prime}} n_{x}{ }^{\prime} \delta_{y^{\prime}} n_{y^{\prime}} \\
& \left.H_{l h}=\left[E_{z}^{l h}+\hbar c_{0}^{l h} \sqrt{\gamma_{1}-\gamma_{2}}\left(\left(n_{x}+n_{y}\right)+A\left(n_{x}-n_{y}\right)+1\right)\right)\right] \delta_{n_{x}, n_{x}{ }^{\prime} \delta_{y^{\prime}} n_{y}^{\prime}}
\end{aligned}
$$

The off diagonal terms are also calculated using the expression of $b$ \& $c$ [19]. 


\section{RESULTS AND DISCUSSIONS}

Numerical calculations have been carried out to find the size and shape dependence of electronic structure of GaAs/AlGaAS QDs. The parameters used in these calculation are, $\mathrm{m}_{\mathrm{e}}=0.0665 \mathrm{~m}_{0}$, Luttinger parameters and band gap energy for GaAs are [20-21] $\gamma_{1}=6.85, \gamma_{2}=2.1, \gamma_{3}=2.9$.

Fig.(1) shows the variation of hole energy values as a function of shape anisotropy for the case when band mixing is neglected for quantum dot (QD) size equal to $5 \mathrm{~nm}$. It is apparent from the figure that first excited state of hh and lh are degenerate. Hole energy varies linearly with the anisotropy and variations are large enough that various excited states are crossing each other, this crossing depends on anisotropy A and on confinement strength along z- direction i.e. dot size and band offset.

Effect of band mixing on energy states is shown in Fig. (2), we observe that mixing effect removes degeneracy and slightly red-shifts the energy levels. By comparing Fig.(1) and Fig.(2), we can clearly see that the separation between energy levels in Fig.(2) is less in comparison with the separation of energy levels in Fig.(1).

We have also calculated the contribution of different $\mathrm{lh}$ and hh state to a particular mixed state as designated by $\mathrm{G}_{1}-\mathrm{G}_{4}$

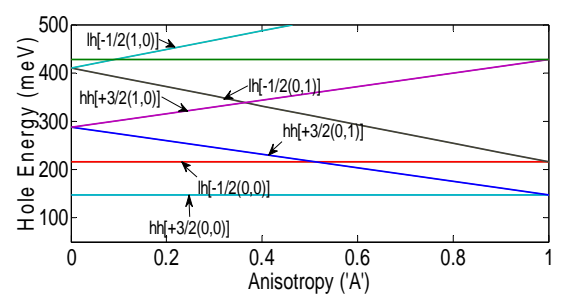

Figure 1: Variation of Hole Energy Eigenvalues as a Function of Anisotropy Parameter (A) in

GaAs/AlGaAs QD, when the effect of Valence Subband Mixing is Neglected

in Fig.(2) using detailed analysis of equivalent eigen vectors is given below in Table 1 .

Table 1

\begin{tabular}{|c|c|c|c|}
\hline \multicolumn{3}{|c|}{ Mixed state } & $\begin{array}{c}\text { Major contributing } \\
\text { states }\end{array}$ \\
\hline $\mathrm{G}_{1}$ & $\mathrm{hh}[-3 / 2(0,0)] ;$ & $\mathrm{hh}[-3 / 2(0,1)] ;$ & $\mathrm{lh}[-1 / 2(0,1)] ; 4.07 \%$ \\
& $87.79 \%$ & $1.85 \%$ & \\
\hline$G_{2}$ & $\mathrm{hh}[-3 / 2(0,0)] ;$ & $1 \mathrm{~h}[-$ & $\mathrm{lh}[-1 / 2(0,1)] ; 49.81 \%$ \\
& $6.30 \%$, & $1 / 2(0,0)] ; 10.73 \%$ & \\
\hline$G_{3}$ & $\mathrm{hh}[-3 / 2(0,0)] ;$ & $\mathrm{lh}[-$ & $\mathrm{lh}[-1 / 2(0,1)] ; 2.58 \%$ \\
& $12.76 \%$ & $1 / 2(0,0)] ; 57.2 \%$ & \\
\hline$G_{4}$ & $\mathrm{hh}[-3 / 2(0,1)] ;$ & $\mathrm{lh}[-$ & $\mathrm{lh}[-1 / 2(0,1)] ; 3.05 \%$ \\
& $21.02 \%$ & $1 / 2(0,0)] ; 50.96 \%$ & \\
\hline
\end{tabular}

enersy Figure 2: Variation of
of Anisotropy Param

Figure 2: Variation of Hole Energy Eigenvalues as a Function of Anisotropy Parameter (A) in GaAs/AlGaAs QD, when Band Mixing Effect is Considered

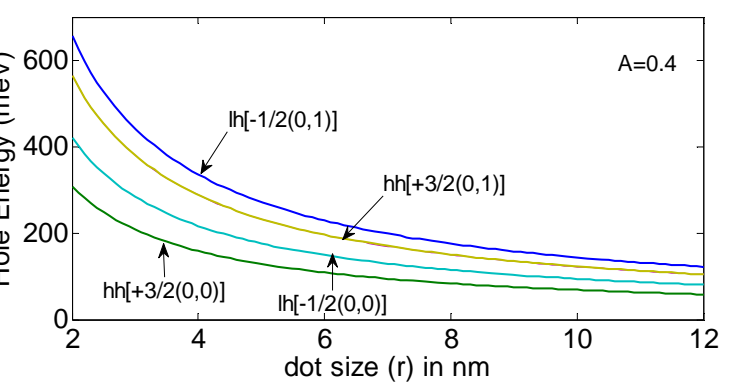

Figure 3: Energy verses Quantum Dot Size when Anisotropy $\mathrm{A}=0.4$.

Fig.(3), shows valence sub-band energies as a function of dot size, figure shows that decrease in size leads to a significant increase in hole energy, this feature clearly exhibits quantum confinement effect. It is also observed from figure that energy separation between various energy states decreases by increase in the dot size because energy splitting of hh and

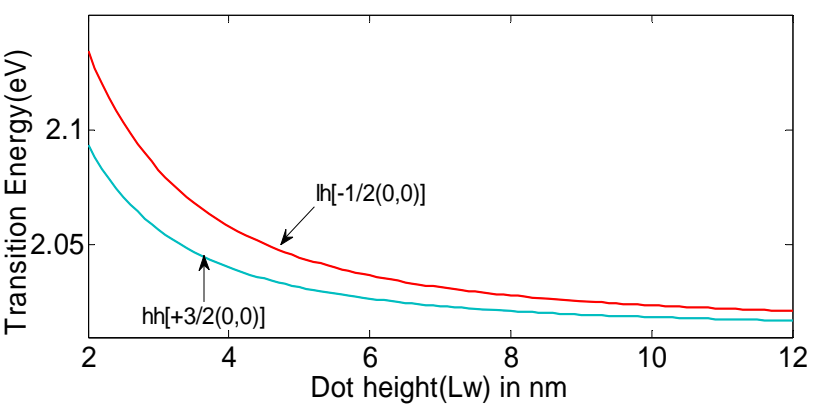

Figure 4: Energy of Quantum Dot as a Function of Dot Height (Lw) confinement along $z$ direction figure also shows that the energy separation between $\mathrm{hh}$ and $\mathrm{lh}$ decreases with the increase in height because as height increases confinement decreases so energy splitting of hh and lh decreases 


\section{CONCLUSION}

We have analyzed the effect of size and shape on electronic structure of QDs. The energy states without mixing case have a significant variation with anisotropy as compared to with mixing case and higher energy states show considerable change but ground states show slight variation with anisotropy. Sub-band mixing effect leads to the significant change in energy levels, this effect is visible in terms of removal of degeneracy and red-shifting of energy levels. Hole energies and transition energies show considerable quantum confinement effect, which manifest in blue shifting of energy levels with reduction in QD size. Separation between valence sub-band energy levels also increases with the decrease in QD size and height. We can conclude here that size, shape anisotropy and mixing have remarkable effect on electronic structure of QDs.

\section{REFERENCES}

[1] J.J. Coleman, IEEE J. Quantum Electron. Vol. 6, 1008, 2000.

[2] J. Faist, Nat. Photonics Vol. 3, 11, 2009.

[3] F. Qian, S. Gradec ak, Y. Li, C.Y. Wen and C.M. Lieber, Nano. Lett. Vol. 5, 22872005.

[4] O.K. Andersen and O. Jepsen, Phys. Rev. Lett. Vol. 53, 25711984.

[5] G. Jolley, L. Fu and H.H. Tan et. al. Appl. Phys. Lett. Vol. 92, 193507, 2008.

[6] S. Sanguinetti, K. Watanabe,T. Kuroda and F. Minami et. al. Journal of crystal growth, Vol. 242, Pp.321-331, 2002.

[7] I. Kegel et al., Phys. Rev. Lett. Vol. 85, 1694, 2000.

[8] F. Boscherini et al., Appl. Phys. Lett Vol. 76, 682, 2000.

[9] U. Denker, M. Stoffel, and O. G. Schmidt, Phys. Rev.Lett. Vol. 90, $196102,2003$.

[10] O. Gunawan, H.S. Djie, and B.S. Ooi, Phys. Rev. B, Vol. 71, 205319, 2005.

[11] C.Y. Ngo, S.F. Yoon, W.J. Fan, and S.J. Chua, Phys. Rev. B, Vol. 74, 245331, 2006.

[12] H.T. Jiang and J. Singh, Phys. Rev. B 56, 4696, 1997.

[13] O. Stier, M. Grundmann, and D. Bimberg, Phys. Rev. B, Vol. 59, 5688, 1999.

[14] N. Vukmirovic', D. Indjin, V.D. Jovanovic', Z. Ikonic', and P. Harrison, Phys. Rev. B , Vol. 72, 075356, 2005.

[15] A. Schliwa, M. Winkelnkemper, and D. Bimberg, Phys Rev. B, Vol. 76, 205324, 2007.

[16] S.L. Chuang "Physics of photonic devices" John Wiley \& sons, 1995.

[17] U. Bockelmann and G. Bastard, "Interband absorption in quantum wires. I. Zero-magnetic-field case" Phys. Rev. B, Vol. 45, 1688, 1992.

[18] W.W. Chow, S.W. Koch, and M. Sargent III, Semiconductor Laser Physics, Springer-Verlag, Berlin, Pp. 463-464, 1994.

[19] J. Kumar, S. Kapoor, S.K. Gupta and P.K. Sen Phys. Rev. B, Vol. 74 115326, 2006.

[20] U. Ekenberg, Phys. Rev. B 40, 7714, 1989.

[21] I. Vurgaftman and J.R. Meyer, J. Appl. Phys., Vol. 89, 5824, 2001.

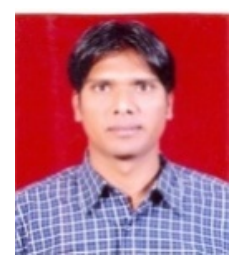

Mr. Dharmendra Kumar received B.Tech from IET MJP Rohilkhand University Bareily, U.P. and Master of Technology (M.Tech) from Indian School of Mines, Dhanbad, Jharkhand, India in area of Electronics \& Communication Engg., in year 2009 and 2011 and is currently pursuing Ph.D. Degree in the department of Electronic Engineering at Indian School of Mines, Dhanbad, Jharkhand, India. His areas of research are Nano-photonics, nanoelectronics, Semiconductor Nanostructure. (Email:dharmendra0127@gmail.com) 